\title{
A Case of Upper Extremity Edema
}

\author{
Leslie Russell ${ }^{\mathrm{a}, \mathrm{c}}$, Kelly McGarry
}

\begin{abstract}
Upper extremity deep venous thrombosis (UEDVT) accounts for the minority of thrombotic events. Most UEDVT events are secondary to venous catheters allowing for easy diagnostic recognition. Paget-Schroetter syndrome (PSS), formation of UEDVT due to mechanical compression of the subclavian vein, is an uncommon cause of primary UEDVT and therefore often remains elusive. Clinicians should maintain a high degree of suspicion for this disorder in young males with spontaneous upper extremity edema as prompt detection can be curative and prevent long term sequelae. We report a case of a healthy young male presenting with Paget-Shroetter syndrome and resultant pulmonary embolus.
\end{abstract}

Keywords: Paget-Shroetter Syndrome; Upper extremity deep venous thrombosis (UEDVT); Pulmonary embolus; Thrombectomy

\section{Introduction}

Upper extremity deep venous thrombosis (UEDVT) accounts for a minority of thrombotic episodes. The vast majority of UEDVT arise secondary to central venous catheters or access devices. Paget-Schroetter syndrome (PSS), formation of venous thrombosis due to mechanical compression of the subclavian vein by anatomic abnormalities or upper chest muscular hypertrophy, is an uncommon cause of pri-

Manuscript accepted for publication June 6, 2013

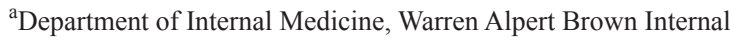
Medicine Rhode Island Hospital, USA

${ }^{b}$ Alpert Medical School at Brown University, General Internal Medicine Residency Program, University Medicine, 593 Eddy Street, DGIM office, Providence, RI 02903, USA

${ }^{\mathrm{c} C o r r e s p o n d i n g ~ a u t h o r: ~ L e s l i e ~ R u s s e l l, ~ B r o w n ~ I n t e r n a l ~ M e d i c i n e ~ P G Y 2 ~}$ Resident, 26 Grotto Ave Apt 1R, Providence, RI 02906, USA.

Email: Lrussel1906@gmail.com

doi: http://dx.doi.org/10.4021/jmc1335w mary UEDVT. Typically occurring in young, healthy males, the diagnosis often goes unrecognized and can result in serious complications if overlooked. Here we report a case of Paget-Schroetter syndrome resulting in pulmonary embolus.

\section{Case Report}

A 20-year-old man presented to the emergency department with three days of swelling and erythema of the right upper extremity. There was no history of trauma, fever, pain,

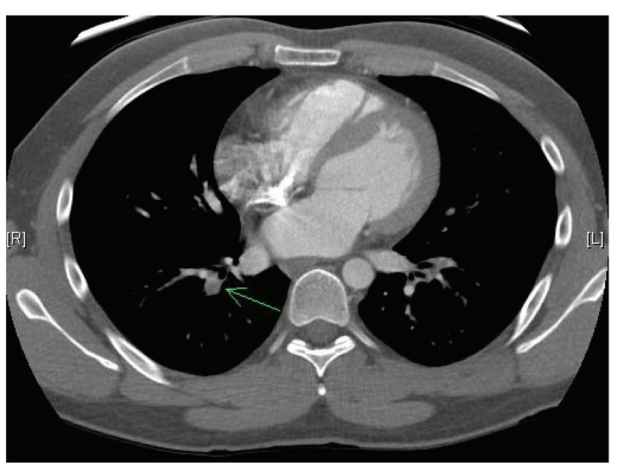

a

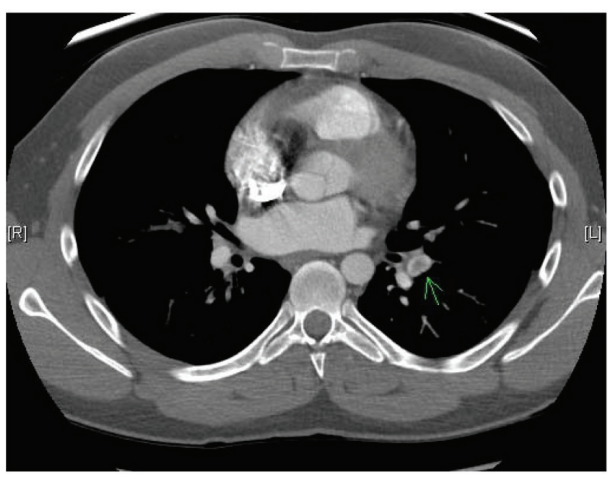

b

Figure 1. $(a, b)$. Green arrows pointing to right and left bilateral pulmonary emboli seen on CT scan. 


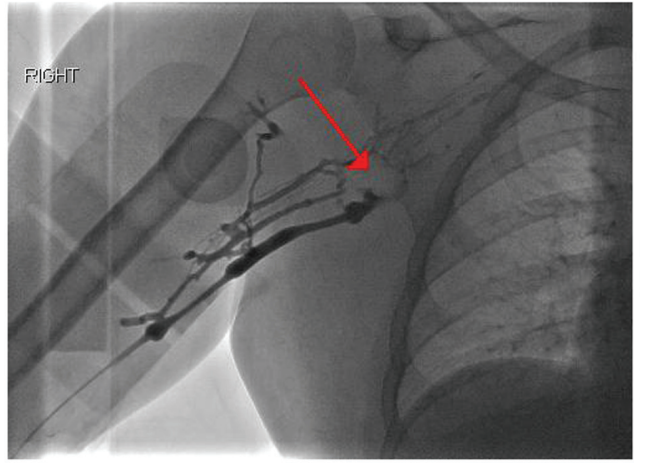

Figure 2. Venography demonstrating occlusive thrombus in the axillary and subclavian veins with multiple collaterals.

or neurologic compromise. Chest x-ray was within normal limits. Venous doppler ultrasonography demonstrated right subclavian and axillary vein thrombosis. CT angiography illustrated bilateral pulmonary emboli (Fig. 1). Systemic anticoagulation with intravenous heparin infusion and oral warfarin therapy was initiated.

Echocardiogram did not demonstrate right ventricular dysfunction. Initial venography demonstrated occlusive thrombus in the axillary and subclavian veins (Fig. 2). The patient underwent mechanical thrombectomy followed by six hours of tissue-plasminogen activator infusion. Post-procedure CT angiography showed reduction of thrombi though persistent stenosis of the subclavian vein at the costoclavicular junction. The patient subsequently underwent first rib resection and scalenectomy for presumed Paget-Schroetter syndrome. Post-procedure CT venogram demonstrated occlusive thrombosis extending from the right axillary vein to the right subclavian vein (Fig. 3). Repeat thrombectomy and thrombolysis led to resolution of thrombus, however there was persistent significant stenosis in the right mid-subclavian vein (Fig. 4). Venoplasty and stenting was performed with successful resolution of stenosis (Fig. 5). The patient was subsequently discharged home on systemic anticoagulation.

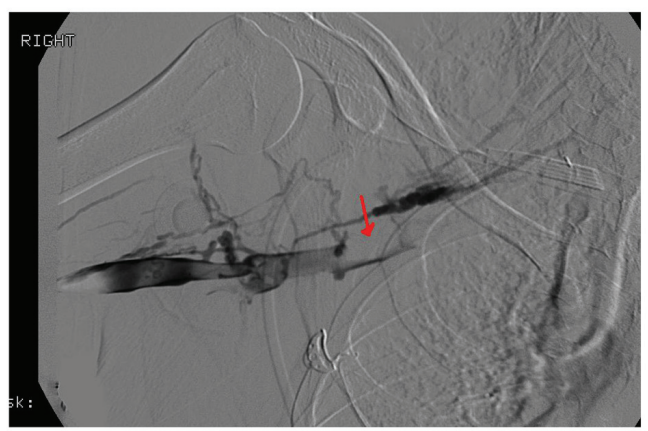

Figure 3. Red arrow documenting occlusive thrombus extending from the right axillary vein to the right subclavian vein on venography.

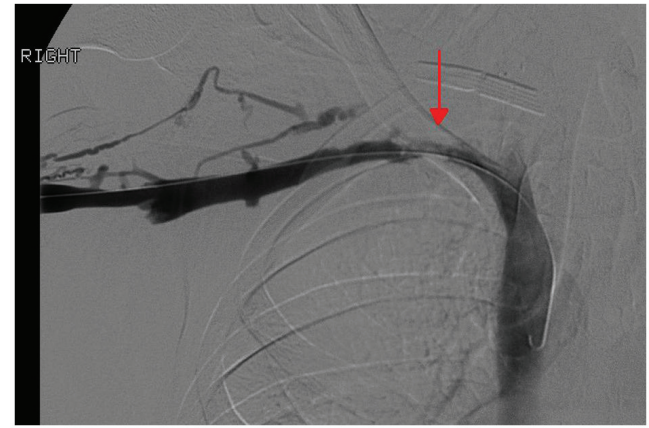

Figure 4. Repeat venography with resolution of thrombus but continued hemodynamically significant stenosis (red arrow) in the mid right subclavian vein.

\section{Discussion}

Paget-Schroetter syndrome is an uncommon cause of UEDVT. The syndrome usually presents in healthy young males as upper extremity edema, pain and erythema. It most commonly occurs in the setting of vigorous activity; rarely, a specific precipitating event cannot be ascertained [1]. In this patient population, aggressive diagnostic evaluation should be performed, as surgical correction of anatomic abnormalities can be curative. Initial diagnostic modality includes venous doppler ultrasonography to detect thrombosis and obstruction. This should be followed by chest CT to detect bony abnormalities. It is imperative to maintain a high degree of clinical suspicion, as often these testing modalities are equivocal. Conventional venoangiography is often necessary as the gold standard for diagnosis [1]. It is also important to assess the contralateral extremity as obstruction frequently occurs bilaterally [2].

Numerous predisposing congenital anatomical anomalies are associated with the syndrome, including cervical ribs, extra ribs, scalene muscle hypertrophy, or abnormal muscle tendon insertion sites [1]. Patients may also develop

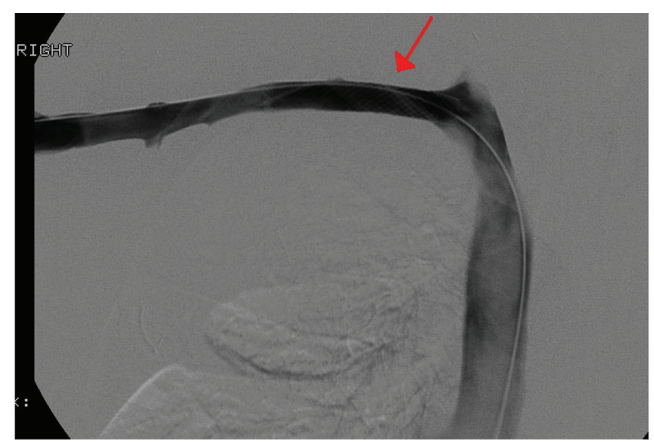

Figure 5. Right upper extremity venogram following Wallstent placement $(4 \mathrm{~cm} \times 12 \mathrm{~cm})$ with resolution of the focal significant stenosis in the right mid subclavian vein. Red arrow pointing to the location of the Wallstent. 
venous compression through repetitive movements, or from bony rib trauma. These movements cause continual venous injury and results in expansive fibrosis and intimal hyperplasia $[1,3,4]$. This fibrosis occurs both within the vein itself as well as around the vessel, causing contraction and venous blood flow stasis [4]. Recurrent injury leads to initiation of the coagulation cascade thrombosis [2,3]. Anatomic abnormalities may also reduce mobility of the subclavian vein, further predisposing to obstruction and thrombosis [1]. In the patient discussed above, his first rib anatomy as well as scalene muscle hypertrophy presumably resulted in venous compression and development of PSS.

Complications of PSS include pulmonary emboli and post-thrombotic syndrome [1]. Post thrombotic syndrome develops from thrombosis induced venous valvular injury resulting in valvular incompetence [5]. It can lead to chronic venous insufficiency, pain, edema, and hyperpigmentation [5]. Approximately 10 of patients with PSS develop pulmonary embolus [6].

Management of this complex disorder requires a multidisciplinary and should include Internists, Thoracic Surgery, and Interventional Radiology. Consultation with a Hematologist is often helpful for ruling out alternative thrombophilias. Conventional systemic anticoagulation is a cornerstone of initial therapy. In order to reduce the risk of post-thrombotic syndrome, definitive thrombotic therapy is often necessary utilizing both mechanical thrombectomy and pharmacologic thrombolysis [7]. Definitive surgical correction of the underlying anatomic abnormality is critical to prevent recurrence $[4,6]$. The timing of these procedures however remains controversial [3]. Long term anticoagulation is usually required for at least three months [3].

While Paget-Shroetter syndrome remains an uncommon cause of UEDVT, clinicians should maintain a high degree of clinical suspicion for this elusive syndrome in young, healthy patients with upper extremity edema. This is particularly important in patients presenting with history of repetitive movements who are thought to have musculoskeletal injury alone. Accurate diagnosis can result in prevention of pulmonary embolus or post-thrombotic syndrome and its' associated morbidity. Surgical therapy of anatomic abnormalities can lead to definitive cure, making this challenging disorder rewarding for patients and clinicians alike.

\section{Conflict of Interest}

None.

\section{Grant}

None.

\section{References}

1. Alla VM, Natarajan N, Kaushik M, Warrier R, Nair CK. Paget-schroetter syndrome: review of pathogenesis and treatment of effort thrombosis. West J Emerg Med. 2010;11(4):358-362.

2. Koury JP, Burke CT. Endovascular management of acute upper extremity deep venous thrombosis and the use of superior vena cava filters. Semin Intervent Radiol. 2011;28(1):3-9.

3. Engelberger RP, Kucher N. Management of deep vein thrombosis of the upper extremity. Circulation. 2012;126(6):768-773.

4. Thompson RW. Comprehensive management of subclavian vein effort thrombosis. Semin Intervent Radiol. 2012;29(1):44-51.

5. Kahn SR, Ginsberg JS. Relationship between deep venous thrombosis and the postthrombotic syndrome. Arch Intern Med. 2004;164(1):17-26.

6. Thompson JF, Winterborn RJ, Bays S, White H, Kinsella DC, Watkinson AF. Venous thoracic outlet compression and the Paget-Schroetter syndrome: a review and recommendations for management. Cardiovasc Intervent Radiol. 2011;34(5):903-910.

7. Papantoniou E, Morgan-Rowe L, Johnston E, Brennand D, Raja J, Hague J. Pharmacomechanical thrombolysis in the management of paget-schroetter syndrome. Case Rep Radiol. 2013;2013:214804. 